A pre and post session confidence questionnaire was provided, and participants were asked to share additional comments.

Results 15 nurses and 25 paediatric doctors were trained in using the pigtail chest drain. $100 \%$ of participants reported feeling more confident with this technique following the training. One registrar has since successfully inserted a pigtail chest drain for a neonate with a large pneumothorax.

5 nurses and 20 paediatric doctors were trained in using the Midline, with all participants feeling more confident with this technique following the training. Four midlines have been successfully inserted into patients requiring prolonged antibiotic regime following these sessions. Three midlines lasted their intended time length, with no complications, and meant patients did not have to return to hospital for repeat vascular access. One midline was dislodged in the context of multiple blood sampling.

Conclusions Following its success we intend on ingraining these sessions within the trainee-teaching curriculum. For clinicians wanting to utilise their skill on real patients, the consultants will supervise this, as required. All procedures will be logged, so they can be audited for quality control. We recommend all paediatric DGH settings incorporate these skills into their units to enable safer and better patient care, and trainee skill-set. However, we recognise the variability in available expertise in all units to undertake training.

\section{British Society for Paediatric Dermatology}

\section{MUCO-CUTANEOUS MANIFESTATIONS IN CHILDREN WITH THALASSEMIA: AN OBSERVATIONAL STUDY}

${ }^{1}$ Ashna Kumar, ${ }^{2}$ Masarat Jabeen, ${ }^{2}$ Preeti Sharma. 'LHMC New Delhi, India; ${ }^{2}$ GMC Jammu, India

\subsection{6/archdischild-2021-rcpch.123}

Background Thalassemia syndromes are a group of autosomal recessively inherited single gene haemoglobinopathies. Beta thalassemia major affects almost every organ system of the body. Several studies have been reported on various systemic complications of beta-thalassemia major, but there is scarce data regarding the muco-cutaneous manifestations of this disease. This study was conducted to describe the muco-cutaneous manifestations noted in multi-transfused beta-thalassemia major children and thus evaluate their correlation with iron overload

Objectives To describe the muco-cutaneous manifestations seen in children living with thalassemia major

Methods A descriptive observational cross-sectional study was conducted on 68 diagnosed patients of thalassemia major attending the thalassemia unit for transfusion at a tertiary care hospital. The data was collected by direct interview of the patients receiving blood transfusion at the thalassemia unit and by a thorough examination of the skin, hair, nails and oral mucosa of these patients.

Results The study included 68 children in the age range of 6 months to 19 years with an average age of the enrolled children being 10.5 years and with a male: female ratio of $1.72: 1$. The common muco-cutaneous manifestation seen in these patients included knuckle hyperpigmentation (60.2\%), moderate pallor (42.6\%), icterus (26.4\%), lusterless hairs (20.5\%), leuconychia striata $(14.7 \%)$, and oral ulcers (10.2\%).

Conclusions All thalassemic children had atleast one cutaneous manifestation. Thus, careful muco-cutaneous evaluation is required in multi-transfused thalassemics to provide an early diagnosis of dermatological diseases in them. Dermatological evaluation is a valuable part of thalassemia care and hence should be carried in every child suffering from thalassemia.

\section{British Society for Rheumatology}

\section{MACROPHAGE ACTIVATION SYNDROME AND SEPSIS}

Ashna Kumar. LHMC New Delhi, India

\subsection{6/archdischild-2021-rcpch.124}

Background Sepsis is a state of systemic inflammation due to an infectious etiology leading to multisystem dysfunction and death in severe cases. The morbidity and mortality rises further due to an underlying immuno-modulatory process presenting as MAS (Macrophage Activation Syndrome) than accompanies the state of sepsis.

Objectives To describe a case series of three infants with hyper-ferritinemia and MAS being successfully treated with less immunosuppressive and cytotoxic methylprednisolone and IVIg therapies.

Methods Three children presenting with clinical symptoms and signs of sepsis being treated with antibiotics but not responding to the conventional treatment were screened for the biomarkers of inflammation and satisfied the criteria for Macrophage Activation Syndrome and thus treated with immuno-modulatory therapy. this resulted in a satisfactory outcome.

Results The patients were treated with immunomodulatory therapy with IVIg and methylprednisolone. (One of our cases presented in the neonatal period and we avoided methylprednisolone in the patient weighing risks and benefit). Such immune-modulation slows down the inflammatory storm by reducing cytokines and providing pathogen specific antibodies. These modalities of treatment have been shown to be less immunosuppressive and less cytotoxic in patients with hyperferritinemia and MAS(Macrophage Activation Syndrome) than the chemotherapeutic drugs used in HLH (Hemophagocytic Lymphohistiocytosis). The use of these modalities in these cases led to successful outcome in terms of survival of these children presenting as MAS with sepsis.

Conclusions In setting of sepsis, MAS should be suspected if clinical condition is not responding despite appropriate management or laboratory parameters show progressive thrombocytopenia and transaminitis. Screening for MAS can be easily done by serum investigations like ferritin, fibrinogen, triglycerides, platelet count and liver enzymes. The timely administration of IVIg and methylprednisolone in these children reverses the inflammatory state and prevents irreversible organ damage. Thus addition of these immunomodulatory therapies in addition to antibiotics and supportive care improves the patient outcomes. 